\title{
Imagining Numbers (particularly the square root of minus fifteen)
}

\author{
Reviewed by Steven G. Krantz and Dietmar Dath
}

\author{
Imagining Numbers (particularly the square root \\ of minus fifteen) \\ Barry Mazur \\ Farrar, Straus and Giroux, 2003 \\ 260 pages, $\$ 22.00$ \\ ISBN 0-374-17469-5
}

\section{Steven G. Krantz}

Harvard University, founded in 1636, is America's oldest institution of higher learning. It is the wellspring of many of our intellectual traditions. One of my favorite of these is the ritual of various Harvard faculty from the humanities and the sciences and the social studies getting together once per month or so to exchange ideas. It is a fascinating exercise: the humanist trying to explain to the cosmologist the current issues of deconstructionism; the homotopy theorist explaining to the philologist about toposes; the philosopher informing the geneticist about logical positivism. Barry Mazur is evidently the product of this crucible of erudition. His work, obviously a popular math book, is not the mindless gibbering of 1089 and All That [ACH], nor is it the self-important bombast of Chaos [GLE]. Barry Mazur has a mission: he wishes to explain to a humanist or a social theorist or a poet what $\sqrt{-15}$ is.

This is a remarkable quest, and I am quite sure that I do not know how to carry it out myself. Bear

Steven G. Krantz is professor of mathematics at Washington University in St. Louis. His email address is sk@math. wust1. edu.

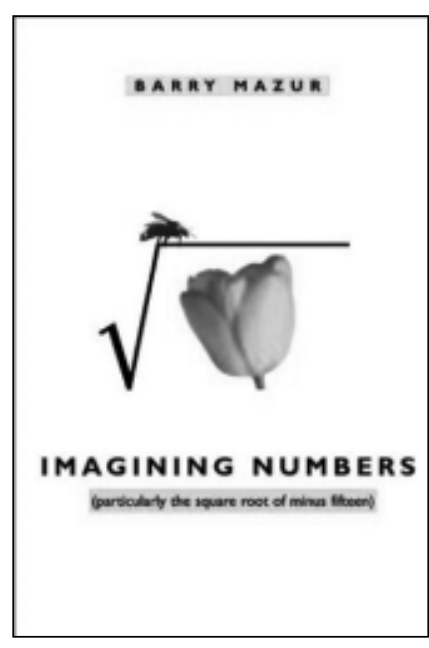

in mind that I am a professional mathematician, an accomplished expositor, and in fact I am a complex analyst. I am supposed to know what $\sqrt{-15}$ is. But in fact I do not. The casual reader might conclude that this is what is wrong with the tenure system: Irresponsible faculty who are accountable to nobody. But that is not really

the nub of the matter.

I have written scores of papers and perhaps half a dozen books on complex analysis. For me the complex numbers are a device, or a trick if you will, for giving the Euclidean plane a field structure. Nothing more nor less. (They are also an interesting source of geometries, but that fact is not salient to the present discussion.) If $\sqrt{-15}$ were to disappear from the face of the earth tomorrow, it would not affect my life one jot. In fact, I could give up countably many of the complex numbers and happily continue to write my books and papers. My guess is that your typical humanist or social theorist or poet has even less use for $\sqrt{-15}$ than do I. And less motivation to find out more about it. Even so, it is an interesting (if a bit effete) intellectual challenge to determine how to make the 
idea of $\sqrt{-15}$ accessible to someone with no training.

I think that most of us do not fully comprehend the magnitude of the task that Barry Mazur has set for himself. I was once chatting with a humanist who told me that if he is ever at a talk and the presenter exhibits a graph or a table or a histogram or a pie chart, then his mind simply shuts down. We are not talking here about people who are simply uninformed about quantitative thinking. Rather, we are talking about people who revile quantitative thinking. How does one get through to them? Mazur seems to know.

The real question then is, does Mazur succeed? Mazur is a smart guy, and he has a good sense of his audience. He realizes that his poet probably does not merely have a problem conceptualizing $\sqrt{-15}$; in point of fact he probably does not even know what a square root-for example $\sqrt{4}-$ actually is. So Mazur begins at the beginning. In fact, like Alfred Jules Ayer, Mazur begins by pondering how ideas travel from the printed page to the brain. He moves on to the elementary concepts of number and square root, illustrating them with (among other things) the quadratic formula. He discusses negative numbers and why a minus times a minus is a plus. As one who has struggled with communicating mathematics to the great unwashed, I must say that I admire Mazur's courage and tenacity and patience.

One of the charming features of Mazur's book is that it treats the reader to a considerable, if episodic, amount of mathematical history and culture. We are introduced along our journey to Cardano and Bombelli and Tartaglia and Argand and de Moivre (among many others). We learn about cartesian geometry and how algebraic concepts may be visualized using plane geometry. For the illuminati, we now have a hint of how Mazur will explain the idea of $\sqrt{-15}$ : he will draw a picture of it!

Mazur takes great pains to communicate with his putative humanist in language that such a scholar will appreciate. He sometimes falls thereby into an apologetic mode. The following passage illustrates both points:

Along similar lines, it is tempting to modify Oscar Wilde's assertion that "the morality of art consists in the perfect use of an imperfect medium" to say that the ideal of mathematics, perhaps never achieved, is to do "perfect work in a perfect medium."...I don't believe that last statement, not for a second. The real work of mathematics is hardly characterized by perfect tenses, or imperfect ones.
Throughout the book we are treated to passages from and interpretations of and homilies to Auguste Rodin, Elaine Scarry, Rainer Rilke, Socrates, Shakespeare, Jeremy Bentham, Wordsworth, Quintilian, Eva Brann, John Ashbery, and Geoffrey Chaucer-and that is just in the first twenty-two pages! Perhaps all these allusions will make the putative humanist comfortable. Perhaps this is the argot in which the literary critic or the philosopher is accustomed to communicate. Since I am primarily interested in the mathematics, I find all this pandering to be tiresome. That is perhaps a function of my Weltanschauung.

But the bottom line is whether Mazur finally gets to his point. And in fact he does. On page 145 he begins to discuss $\sqrt{-1}$, and he spends the next eighty pages helping the reader to become accustomed to the idea. For a mathematician this whole process is astonishing, for the mathematical scholar could (starting from scratch) define-in fact construct-the number $\sqrt{-1}$ in less than a page, and he would of course omit that eighty pages of helping the reader to become acclimated. Barry Mazur has had to, in effect, reinvent the way that mathematics is communicated. Imagining Numbers is a remarkable polemic.

Is Mazur's book a success? I think that the answer is "yes", provided that we can find a humanist with the patience and interest to read it. Certainly James R. Newman's The World of Mathematics $[\mathrm{NEW}]$ is far too recondite and unfocused to help the virtually mathphobic literary critic to see what mathematicians do. Many another effort, such as $[\mathrm{ACH}]$, is just too silly. Mazur has the great advantage of knowing his audience, being acquainted with his audience, and having the confidence and skill to actually address that audience. I think that Imagining Numbers is a valuable addition to our literature and can only help to bridge the dreadful chasm that C. P. Snow described in The Two Cultures [SNO]. Another reviewer might say that he hopes that Mazur's effort will inspire other writers to attempt books of the same kind. I have a different hope, and it is that other writers, and other mathematicians, will read Mazur's book and ponder what he has attempted, what it means, and why he did it. It is an important effort, and it has enriched us all.

\section{References}

[ACH] D. ACHESON, 1089 and All That, Oxford Univ. Press, Oxford, 2002.

[GLE] J. GLEICK, Chaos: Making a New Science, Penguin USA, New York, 1998.

[NEW] J. R. Newman, The World of Mathematics, Simon and Schuster, New York, 1956.

[SNO] C. P. SNOw, The Two Cultures, Cambridge Univ. Press, Cambridge, 1993. 


\section{Dietmar Dath}

When we were both twelve years old, one of my closest friends revealed to me what she thought of as the fundamental difference between mathematics and all the other subjects we were taught at school: "In math, when you explain things, you never reach a bottom line where someone says: 'Go no further, this is just the way things are, there's no use for your understanding from here on in.' All the concepts, procedures, and branches of mathematics sustain and explain each other; it's all connected. Above all, this means that you needn't learn a lot of stuff by heart-if you can remember just some of the definitions and axioms, you will eventually be able to deduce and/or reconstruct everything else."

At the time, my friend was waging her own personal vendetta against subjects such as French grammar and zoology, where quite a lot had to be taken on faith and learned by heart. What she had inadvertently stumbled upon when thinking about the advantages of mathematics over other areas of knowledge was the metamethodological idea of holism: in the realm of purest thought, ideas reinforce each other's validity through interdependence and interconnectedness. She would have loved logic, set theory, categories, toposes, and similar topics of fundamental research.

Although we have long since been out of touch, leaving me without the slightest guess as to what became of her liking of mathematics, I suspect she would have found Barry Mazur's book Imagining Numbers (particularly the square root of minus fifteen) appealing to her acquired tastes, for the way that working mathematician Mazur introduces concepts, transformations, and correspondences connected to imaginary and complex numbers throughout his book relies on just such a knack for holism. This starts with his use of relationshipssome of them discovered rather late in the history of the field-between number theory and geometry, yet it does not stop here, for one of Mazur's aims is to demonstrate the applicability of the holist idea to the very connection between mathematical and nonmathematical areas of knowledge and creativity.

Underlying this, the reader can easily discern a very earnest quest for, as we say in German, $A n$ schaulichkeit-this is one of those existential and essentially untranslatable words with which the language of Gauss and Einstein is either blessed or burdened, depending on your point of view. It basically means "the quality (of a concept) to be apparent to the naked eye" but in a metaphorical

Dietmar Dath is an editor at the Frankfurter Allgemeine Zeitung. His email address is d. dath@faz. de. sense that is not referring to the faculty of vision (if you can bring yourself to believe that).

By drawing a fine-yet, in everyday experience, somewhat blurry-distinction between "to imagine" and "to picture", Mazur wishes to point out that such Anschaulichkeit is not so much an attribute of ideas like the imaginary quantities themselves but rather a measure for the individual mind's ability to do something with those ideas, to make use of them creatively. His argument thus starts with reflections on counting, relates the square root of a number to the geometrical notion of a square, and then proceeds to relate the not-so-rational numbers to ratios.

Explaining the unknown by way of the known is his master plan, plucking numerous quotations in both verse and prose from Shakespeare and Rilke, ancient Indians, and recent Americans along the way. The reader is presented with brief glimpses of significant moments in the history of imaginary numbers, which are intermittently juxtaposed with more general musings on how we as human beings learn, how we come up with new problems and solutions, and so forth. Mazur switches between narrative, expository, and speculative passages, which works comfortably most of the time. The tone of the text is reassuring, inviting, even enticing, if at times in danger of becoming a bit too cutesy, but never of talking down to the reader.

Yet Mazur stays in control of his subject matter throughout. Even seemingly ornamental touches-the book is richly illustrated not just with schematics but also with the odd photograph and even a drawing of Vladimir Nabokov's-add to the genuine sense of intellectual drama, shining through especially vividly in the chapter on "Bombelli's puzzle", Mazur's most accomplished historical excursion.

Some readers might get irritated by the large amount of footnote material, but judging from experience with this sort of book, excessive quoting seems an occupational hazard for writers who, starting from some subject in the exact sciences, wish to cross over into the territory of the humanities (most commonly psychology and philosophy) and therefore feel they have to quote other people a lot. Whereas primers in the exact sciences usually appeal to your object knowledge, the average poet or philosopher likes a kind of erudition that brings with it a temptation to drop names of the Mighty Dead.

Another point where Mazur might be criticized is that by trying to keep an open mind-namely, the reader's-he sometimes proposes ideas about ideas which might turn out to be truisms, as in the passage where he clarifies the notion of a problem by distinguishing it from a mere question: questions often presuppose answers, whereas problems do not always imply solutions. It sounds striking, to 
be sure, but the problems that truly call for this distinction because they cannot be phrased as questions are certainly exceedingly rare.

Interestingly enough, the didactic approach of emphasizing mathematical imagination over exactitude or what might be called the algorithmic properties of the mathematician's mind lead Mazur to a kind of tongue-in-cheek quasi-intuitionism: he implicitly appeals to intuition almost all the time but does not explicitly grant it any verificatory power that might judge whether a given concept was "righteously" or at least justifiably introduced into the given scientific vocabulary. This recalls the confession once made by Gregory Chaitin: "As a creative mathematician I certainly sympathize with the point of view that the imagination to change the rules of the game is more important than grinding out the consequences of a given set of rules."

A book that is written for the express purpose of popularizing the science, art, and technique that make up mathematics, that touches on such philosophical-i.e., epistemological-aspects of the field might seem odd to many a nonmathematician. To others it might just be a hint at the fact that holism prevails, as inside mathematics so outside of it, a reminder of the very beauty of knowledge-architecture that young minds, or inquisitive minds of any biological age, so often find appealing when mathematics beckons them. 